\title{
11-Dehydro-Thromboxane B2 Measurement
}

National Cancer Institute

\section{Source}

National Cancer Institute. 11-Dehydro-Thromboxane B2 Measurement. NCI Thesaurus.

Code C103344.

The determination of the amount of the 11-dehydro-thromboxane B2 in a sample. 